\section{Estudio descriptivo de mortalidad en sobrevivientes de tortura y prisión política en el período de la dictadura militar en Chile, 1973-1990}

\author{
MARÍA JOSÉ JORQUERA ${ }^{1, a}$, CARLOS MADARIAGA², \\ MARÍA SOLEDAD BURRONE ${ }^{3}$, ERIC TAPIA ${ }^{4, b}$, \\ LISANDRO D. COLANTONIO ${ }^{5}$, RUBÉN ALVARADO ${ }^{6}$
}

\section{Mortality in survivors of torture ad prison during the chilean dictatorship between 1973 and 1990}

Background: Studies on the long-term consequences of torture in survivors in Chile have only addressed the consequences for mental health, leaving aside the physical consequences. Aim: To report the causes of death in the universe of victims of the Chilean civic-military dictatorship recognized by the Reports of the National Commission for Political Prison and Torture (CNPPT) and the Advisory Commission for the qualification of Disappeared Detainees, Politically Executed individuals and Victims of Political Prison and Torture. Material and Methods: The causes of death, age at the time of death, sex, political context of death and opportunity of repair up to June 2016 are described in 38,254 victims of the Chilean civic-military dictatorship. Results: Of the universe of 38,254 victims, 9,152 (23.9\%) died until June 2016. The median age at death was 68 years. The main causes of death were malignant tumors in $28 \%$, cardiovascular diseases in $27 \%$, respiratory diseases in 10\%, digestive diseases in $9 \%$ and external causes in $8 \%$. Conclusions: These results can inform prevention and treatment strategies for victims of the Chilean dictatorship.

(Rev Med Chile 2020; 148: 1773-1780)

Key words: Mortality; Cause of Death; Chile; Survival Rate; Torture.

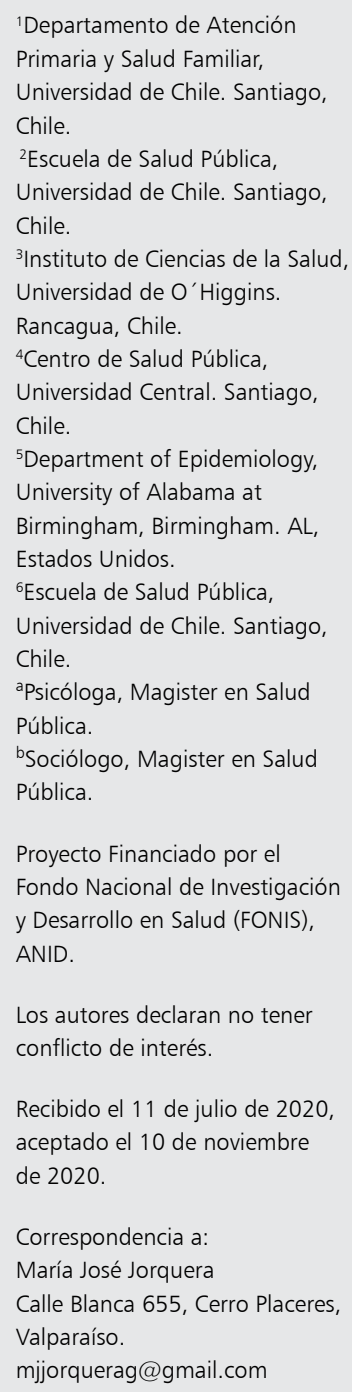

$\mathrm{D}$ iferentes estudios han demostrado que las poblaciones expuestas a estresores físicos y psicosociales extremos presentan, años después, mayores tasas de morbilidad y mortalidad. Este fenómeno se ha observado tanto en prisioneros, militares y población civil en situaciones de guerra y otros conflictos armados ${ }^{1-5}$ como en sobrevivientes del Holocausto ${ }^{6-8}$.

La práctica sistemática de la tortura durante la dictadura cívico-militar chilena (1973-1990) es un ejemplo paradigmático de exposición a traumatización extrema ${ }^{9,10}$. En el año 2005 el Informe de la Comisión Nacional sobre Prisión
Política y Tortura (CNPPT) describió en detalle los métodos de tortura aplicados (dislocación de las articulaciones, aplicación de electricidad, violencia sexual, etc.), los cuales estaban destinados a quebrantar la resistencia física, emocional y moral de las víctimas. De acuerdo la CNPPT, la ausencia de atención oportuna y estrategias integrales de reparación debido al contexto represivo de la época, pudieron haber favorecido la aparición de secuelas en el largo plazo ${ }^{11}$.

La presión política ejercida por diversas organizaciones sociales de víctimas hizo que en el año 2003 el gobierno crease la CNPPT con la misión 
de "determinar, [...] quiénes son las personas que sufrieron privación de libertad y tortura por razones políticas" ${ }^{\prime 1}$. Para ser reconocido como víctima, esta comisión estableció, según el Decreto Supremo (Interior) No 1.040, tres criterios: a) que la privación de libertad y/o tortura fueron perpetradas por razones políticas; b) que los torturadores hayan sido agentes del Estado o personas a su servicio; $y$ c) que los hechos se hubieran cometido entre el 11 de septiembre de 1973 y el 10 de marzo de 1990. En el caso de las personas fallecidas, se autorizó a los familiares directos para que presenten sus testimonios y acrediten las experiencias de prisión y/o tortura. Tanto en el caso de las denuncias de sobrevivientes como en las denuncias de familiares directos, la información se contrastó con otras fuentes de datos para su validación, incluyendo Organismos del Estado, Organizaciones de Derechos Humanos y otras Organizaciones Internacionales ${ }^{11}$. Finalmente, el Estado de Chile, a través de la CNPPT (que funcionó en dos períodos, 2004/2005 y 2010/2011, el último bajo la denominación de Comisión Asesora para la calificación de Detenidos Desaparecidos, Ejecutados Políticos y Víctimas de Prisión Política y Tortura), reconoció oficialmente a 38.254 personas como víctimas de la dictadura cívico-militar ${ }^{11,12}$.

Los testimonios dados a la CNPPT permitieron identificar múltiples consecuencias inmediatas y tardías de la tortura, siendo las más frecuentes: secuelas sensoriales, osteomusculares, genitales y neurológicas; dolor crónico; alteraciones emocionales y conductuales; empobrecimiento de las relaciones sociales y afectivas; distorsiones de la significación y el ejercicio de la sexualidad; rupturas familiares; problemas laborales y empobrecimiento económico; enfermedades mentales diversas; muerte prematura ${ }^{11}$. El objetivo del presente estudio fue describir las causas de muerte en el universo de las víctimas de la dictadura cívico-militar chilena, reconocidas por los Informes de la Comisión Nacional de Prisión Política y Tortura (CNPPT) y la Comisión Asesora para la calificación de Detenidos Desaparecidos, Ejecutados Políticos y Víctimas de Prisión Política y Tortura.

\section{Material y Método}

Se realizó un estudio de tipo transversal y descriptivo sobre el universo de víctimas reconocidas por el Estado de Chile $(\mathrm{n}=38.254)^{11,12}$. Los datos personales de las víctimas, incluyendo fecha de nacimiento y sexo, fueron obtenidos de la Agrupación de Familiares de Ex Prisioneros Políticos Fallecidos-Chile. La base de datos suministrada por esta agrupación fue creada a través de un convenio con el Registro Civil de Chile. Los datos personales se cruzaron con la base de datos del Departamento de Estadísticas e Información en Salud (DEIS) de Chile para identificar el año de defunción y la causa básica de muerte hasta junio del año 2016.

Por cada persona fallecida se determinó la edad al momento de la defunción usando la fecha de nacimiento. A partir del año de defunción se determinó además el contexto político del deceso y la oportunidad de reparación de las víctimas. En cuanto al contexto político, las muertes ocurridas entre los años 1973 y 1990 se consideraron como producidas en dictadura, y aquellas entre los años 1991 y 2016 como fallecidos en democracia. En cuanto a la oportunidad de reparación, se consideraron como sin oportunidad de reparación las muertes ocurridas hasta el año 2003 (previo a la CNPPT), y con oportunidad de reparación las muertes ocurridas a partir del año 2004. La causa de muerte se clasificó según las categorías y subcategorías de la Clasificación Internacional de Enfermedades y Problemas Relacionados, décima revisión (CIE-10).

Se calculó el porcentaje de fallecidos hasta junio de 2016. Por último, se analizó la distribución de la causa básica de muerte por categorías y subcategorías de la CIE-10. Para describir la edad de fallecimiento se calculó la media y el desvío estándar. Para el análisis descriptivo de las variables categóricas se calcularon porcentajes. Para el análisis se utilizó el programa estadístico IBM SPSS Statistics, versión 25.

Finalmente es importante mencionar que la investigación cuenta con el consentimiento de la Agrupación de Familiares de Ex Presos Político Fallecidos-Chile para publicar los resultados del estudio.

\section{Resultados}

Del total de 38.254 víctimas reconocidas por el Estado, $5.201(13,6 \%)$ fueron mujeres y 33.053 $(86,4 \%)$ hombres. Hasta junio de 2016 se identificaron $9.152(23,9 \%)$ fallecidos sobre el total. 
Esto incluye 753 fallecimientos entre mujeres, lo que representa $14,5 \%$ sobre el total de mujeres y 8.399 fallecimientos entre hombres, lo que representan $25,4 \%$ sobre el total de hombres. La edad promedio de defunción fue 68,5 años, similar entre hombres y mujeres. La mayoría de las muertes $(88,7 \%)$ ocurrieron durante el periodo de democracia. Alrededor de un tercio del total de fallecidos murió sin oportunidad de reparación (antes del año 2004) (Tabla 1).

De las muertes agrupadas de acuerdo a las categorías de la CIE-10, 28,3\% correspondieron a tumores y neoplasias, seguidas por las enfermedades del sistema circulatorio $(27,4 \%)$, enfermedades del sistema respiratorio $(9,6 \%)$, enfermedades del sistema digestivo $(8,8 \%)$ y causas externas de morbilidad y mortalidad $(7,7 \%)$ (Tabla 2 ). Estas cinco categorías aglutinaron $81,8 \%$ de las muertes. En los hombres, las cinco primeras categorías de muerte correspondieron a las mismas categorías observadas en la población general. En las mujeres, las primeras cinco categorías de muerte fueron:
Tabla 1. Características demográficas de las víctimas de la dictadura cívico-militar fallecidas hasta el año 2016

\begin{tabular}{|lcc|}
\hline \multicolumn{2}{|c}{ Características demográficas (n = 9.152) } \\
Sexo & $\mathrm{n}$ & $\%$ \\
Hombres & 8.399 & 91,8 \\
Mujeres & 753 & 8,2 \\
Edad de defunción (años) & $\mathrm{Md}$ & $\mathrm{DE}$ \\
Hombres & 68,3 & 13,0 \\
Mujeres & 70,1 & 13,7 \\
Edad de defunción (tramos) & $\mathrm{n}$ & $\%$ \\
$20-29$ & 56 & 0,6 \\
$30-39$ & 198 & 2,2 \\
$40-49$ & 512 & 5,6 \\
$50-59$ & 1.292 & 14,1 \\
$60-69$ & 2.512 & 27,4 \\
$70-79$ & 2.624 & 28,7 \\
$\geq 80$ & 1.958 & 21,4 \\
Contexto político & $\mathrm{n}$ & $\%$ \\
Dictadura (1973-1990) & 1.030 & 11,3 \\
Democracia (1991-2016) & 8.122 & 88,7 \\
Oportunidad de reparación & $\mathrm{n}$ & $\%$ \\
Sin reparación (1973-2003) & 3.133 & 34,2 \\
Con reparación (2004-2016) & 6.019 & 65,8 \\
& & \\
\hline
\end{tabular}

Tabla 2. Causas de muertes por categorías de la CIE-10

\begin{tabular}{|c|c|c|c|c|c|c|}
\hline \multirow[t]{2}{*}{ Causas generales de mortalidad (Categorías CIE-10) } & \multicolumn{2}{|c|}{ Hombres } & \multicolumn{2}{|c|}{ Mujeres } & \multicolumn{2}{|c|}{ Total } \\
\hline & $\mathbf{n}$ & $\%$ & $\mathbf{n}$ & $\%$ & $\mathbf{n}$ & $\%$ \\
\hline Tumores, neoplasias & 2.373 & 28,3 & 241 & 32,0 & 2.614 & 28,6 \\
\hline Enfermedades del sistema circulatorio & 2.298 & 27,4 & 191 & 25,4 & 2.489 & 27,2 \\
\hline Enfermedades del sistema respiratorio & 809 & 9,6 & 75 & 10,0 & 884 & 9,7 \\
\hline Enfermedades del sistema digestivo & 743 & 8,8 & 44 & 5,8 & 787 & 8,6 \\
\hline Causas externas de morbilidad y mortalidad & 646 & 7,7 & 24 & 3,2 & 670 & 7,3 \\
\hline $\begin{array}{l}\text { Síntomas, signos y hallazgos anormales clínicos y de laboratorio no } \\
\text { clasificados en otra parte }\end{array}$ & 448 & 5,3 & 40 & 5,3 & 488 & 5,3 \\
\hline Enfermedades endocrinas, nutricionales y metabólicas & 404 & 4,8 & 45 & 6,0 & 449 & 4,9 \\
\hline Enfermedades del sistema genitourinario & 190 & 2,3 & 25 & 3,3 & 215 & 2,3 \\
\hline Enfermedades del sistema nervioso & 171 & 2,0 & 24 & 3,2 & 195 & 2,1 \\
\hline Ciertas enfermedades infecciosas y parasitarias & 178 & 2,1 & 13 & 1,7 & 191 & 2,1 \\
\hline Trastornos mentales y del comportamiento & 83 & 1,0 & 18 & 2,4 & 101 & 1,1 \\
\hline $\begin{array}{l}\text { Enfermedades de la sangre y de los órganos hematopoyéticos y } \\
\text { otros trastornos que afectan el mecanismo de la inmunidad }\end{array}$ & 25 & 0,3 & 6 & 0,8 & 31 & 0,3 \\
\hline Enfermedades del sistema osteomuscular y del tejido conjuntivo & 17 & 0,2 & 5 & 0,7 & 22 & 0,2 \\
\hline Enfermedades de la piel y del tejido subcutáneo & 12 & 0,1 & 1 & 0,1 & 13 & 0,1 \\
\hline Malformaciones congénitas, deformidades y anomalías cromosómicas & 2 & 0,0 & 0 & 0,0 & 2 & 0,0 \\
\hline Embarazo, parto y puerperio & 0 & 0,0 & 1 & 0,1 & 1 & 0,0 \\
\hline Total & 8.399 & 100,0 & 753 & 100,0 & 9.152 & 100,0 \\
\hline
\end{tabular}


Tabla 3. Causas de muertes por subcategoría de la CIE-10

\begin{tabular}{|c|c|c|c|c|c|c|}
\hline \multirow[t]{2}{*}{ Causas generales de mortalidad (Subcategoría CIE-10) } & \multicolumn{2}{|c|}{ Hombre } & \multicolumn{2}{|c|}{ Mujer } & \multicolumn{2}{|c|}{ Total } \\
\hline & $\mathbf{n}$ & $\%$ & $\mathbf{n}$ & $\%$ & $\mathbf{n}$ & $\%$ \\
\hline Tumores malignos de los órganos digestivos & 1.019 & 12,1 & 79 & 10,5 & 1.098 & 12,0 \\
\hline Enfermedades isquémicas del corazón & 928 & 11,1 & 52 & 6,9 & 980 & 10,7 \\
\hline Enfermedades cerebrovasculares & 701 & 8,4 & 60 & 8,0 & 761 & 8,3 \\
\hline Enfermedades del hígado & 525 & 6,3 & 21 & 2,8 & 546 & 6,0 \\
\hline Tumores malignos de los órganos respiratorios e intratorácicos & 418 & 5,0 & 38 & 5,0 & 456 & 5,0 \\
\hline Causas de mortalidad mal definidas y desconocidas & 366 & 4,4 & 38 & 5,0 & 404 & 4,4 \\
\hline Diabetes mellitus & 365 & 4,3 & 32 & 4,2 & 397 & 4,3 \\
\hline Otras formas de enfermedeades del corazón & 316 & 3,8 & 37 & 4,9 & 353 & 3,9 \\
\hline Influenza (gripe) y neumonía & 293 & 3,5 & 30 & 4,0 & 323 & 3,5 \\
\hline Tumores malignos de los órganos genitales (ambos sexos) & 291 & 3,5 & 20 & 2,7 & 311 & 3,4 \\
\hline Enfermedades crónicas de las vías respiratorias inferiores & 255 & 3,0 & 24 & 3,2 & 279 & 3,0 \\
\hline Enfermedades hipertensivas & 221 & 2,6 & 23 & 3,1 & 244 & 2,7 \\
\hline Eventos de intesión no determinada & 181 & 2,2 & 9 & 1,2 & 190 & 2,1 \\
\hline Accidentes de transporte & 171 & 2,0 & 8 & 1,1 & 179 & 2,0 \\
\hline $\begin{array}{l}\text { Tumores malignos del tejido linfático, de los órganos } \\
\text { hematopoyéticos y tejidos afines }\end{array}$ & 162 & 1,9 & 14 & 1,9 & 176 & 1,9 \\
\hline Tumores malignos de las vías urinarias & 154 & 1,8 & 15 & 2,0 & 169 & 1,8 \\
\hline Lesiones autoinflingidas intensionalmente & 138 & 1,6 & 4 & 0,5 & 142 & 1,6 \\
\hline $\begin{array}{l}\text { Tumores malignos de sitios mal definidos, secundarios y de sitios } \\
\text { no especificados }\end{array}$ & 131 & 1,6 & 12 & 1,6 & 143 & 1,6 \\
\hline Otras causas & 1.764 & 21,0 & 237 & 31,5 & 2.001 & 21,8 \\
\hline Total & 8.399 & 100,0 & 753 & 100,0 & 9.152 & 100,0 \\
\hline
\end{tabular}

1. Tumores y neoplasias.

2. Enfermedades del sistema circulatorio

3. Enfermedades del sistema respiratorio

4. Enfermedades endócrinas, nutricionales y metabólicas.

5. Enfermedades del sistema digestivo.

La Tabla 3 muestra la distribución de subcategorías de la CIE-10 que explican aproximadamente $80 \%$ de las muertes en la población de víctimas de la dictadura cívico-militar chilena. La subcategoría diagnóstica de mayor prevalencia, tanto en hombres como en mujeres, fue los tumores digestivos. La segunda causa de muerte establece una diferencia entre hombres y mujeres, los primeros mueren por enfermedades isquémicas del corazón $(11,1 \%)$ y las segundas por enfermedades cerebrovasculares $(8,0 \%)$. La tercera subcategoría diagnóstica como causa de muerte en los hombres son las enfermedades cerebrovasculares $(8,4 \%)$ $\mathrm{y}$ en las mujeres las enfermedades isquémicas del corazón $(6,9 \%)$. En el caso de los hombres el cuarto lugar lo ocupan las enfermedades del hígado $(6,3 \%)$ y en las mujeres lo ocupan dos subcategorías, ambas con 5,0\% de prevalencia: los tumores malignos de los órganos respiratorios y las causas de mortalidad mal definidas y desconocidas. Los tumores respiratorios ocupan en los hombres el quinto lugar de prevalencia con 5,0\%. Es importante también destacar que tanto hombres como mujeres comparten una misma prevalencia de muerte por diabetes mellitus $(4,3 \%$ y $4,2 \%$ respectivamente). Respecto a la subcategoría lesiones autoinflingidas intencionalmente existe una diferencia en la proporción entre hombres $(1,6 \%)$ y mujeres $(0,5 \%)$. Hacemos esta mención 
aparte dada la trascendencia que tiene esta forma de morir (suicidio) en personas con antecedentes de traumatización extrema asociada a violaciones a los derechos humanos.

\section{Discusión}

La violencia en todas sus dimensiones es un problema de salud pública. Según la Organización Mundial de la Salud (OMS), en el Informe Violencia y Salud, la violencia colectiva (guerra, terrorismo, etc.) es una causa importante de mortalidad, graves alteraciones y secuelas en salud a largo plazo $^{13}$. Una de las modalidades de la violencia colectiva que impactó transversalmente a América Latina fue el proceso de militarización ${ }^{14}$. El presente trabajo aporta información respecto a los procesos de salud/enfermedad de las personas reconocidas como víctimas de la dictadura cívico-militar en Chile, proveyendo datos descriptivos de las causas de muerte más prevalentes y su caracterización.

Los resultados del presente estudio permiten concluir que las neoplasias ocupan la primera causa de muerte en víctimas de la dictadura cívico-militar chilena de ambos sexos; siendo los tumores malignos de los órganos digestivos la primera subcategoría diagnóstica. Este dato es de particular interés para el Programa de Reparación en Atención Integral en Salud (PRAIS), encargado de brindar atención en salud especializada para las víctimas reconocidas por el Estado, pues se ha tenido la persistente sospecha de una alta frecuencia de cáncer en este grupo. Al respecto, las neurociencias han referido que el estrés crónico por traumatización extrema afecta el sistema inmuno-defensivo, favoreciendo la ocurrencia de cáncer ${ }^{15,16}$. Surge aquí la necesidad de realizar estudios comparativos de las tasas de mortalidad según categorías diagnósticas respecto de la población general como también analizar la esperanza de vida en ambos grupos.

En relación con la distribución de las primeras cinco causas de muerte en esta población $(81,4 \%$ del total) se concluye que todas ellas requieren ser objeto de programas preventivos en salud para los sobrevivientes, aspectos que no han sido abordados por el PRAIS. Lo anterior se ratifica con los resultados del presente estudio que evidencian que las neoplasias están en la primera causa de muerte en la muestra estudiada, mostrando una tendencia distinta respecto a la primera causa de muerte en la población general ${ }^{17}$. Transformando al cáncer en un foco de gran interés frente a personas que han vivido experiencias de traumatización extrema.

En lo relativo a la categoría causas externas de morbilidad y mortalidad, que involucra las llamadas muertes violentas (accidentes, homicidios y suicidios), la diferencia entre hombres y mujeres es sustantiva: $7,7 \%$ y $3,4 \%$ respectivamente. De estas causas, los eventos de intención no determinada representan la segunda causa más prevalente de esta categoría, por lo que estimamos que dentro de este grupo se esconde parte significativa del suicidio.Sospecha fundada ante los resultados de un reciente estudio que se centra en el Estrés Post Traumático Complejo, que si bien no aporta estadísticas de suicidalidad, señala que el DESNOS, debido a la extensión, gravedad y complejidad del proceso evolutivo de este trastorno, congrega un subgrupo sintomático que denominan "alteración en la regulación de emociones e impulsos", que genera una alta vulnerabilidad al suicidio o parasuicidio ${ }^{18}$. En relación al suicidio diferenciado por sexo en la población de estudio, es más frecuente en los hombres que en las mujeres, cuestión que en parte es coherente con el comportamiento estadístico en la población general en $\mathrm{Chile}^{17}$, pero estimamos que debe analizarse además el tipo de influencia diferencial que introduce la experiencia de tortura en ambos casos.

La diferencia significativa en el número de casos (hombres y mujeres) reconocidos como víctimas por el Estado de Chile, tiene una posible explicación, desde una perspectiva de género. En la práctica de tortura político-sexual en contra de mujeres (aunque no exclusiva), se trata para ellas de una experiencia de alta sensibilidad como acontecimiento traumático, pues involucra cuestiones como la dignidad humana, la vergüenza y la culpa, sentimientos que inhibieron a muchas mujeres para concurrir a dar testimonio a la $\mathrm{CN}$ PPT. En esta actitud de las mujeres, se refleja no solo la resistencia al estigma social que implica declararse víctima de violencia sexual en una sociedad patriarcal como la nuestra, sino también el desencanto y la frustración de las expectativas de justicia en una sociedad impune, que ha producido altos costos personales como: privatización del daño, producción de duelos cronificados o 
recurrentes, deterioro de la salud mental y física, retraimiento social ${ }^{19}$.

Diversos organismos internacionales han referido que la violencia sexual ha sido utilizada históricamente de manera intencional contra las mujeres en contextos de guerra u opresión política $\mathrm{y}$, por lo tanto, esta práctica debe ser considerada como parte de una acción sistemática ${ }^{20-22}$. Sin embargo, en la recopilación de los testimonios, la CNPPT no incluyó este antecedente, señalando: "Las entrevistas realizadas por esta Comisión no indagaron expresamente acerca de la violencia sexual ejercida contra las ex presas (...) Casi todas las mujeres dijeron haber sido objeto de violencia sexual sin distinción de edades (...) No obstante, se estima que la cantidad de mujeres violadas es muy superior a los casos en que ellas relataron (...) existen numerosos testimonios de detenidos que señalan haber presenciado violaciones, cometidas en una gran cantidad de recintos de detención" 11 .

Respecto a la variable oportunidad de reparación, hemos querido diferenciar aquellos casos que fallecieron antes de la vigencia de la Ley de Reparación de los que fueron falleciendo con posterioridad a ella. Este dato es muy importante pues de este hecho se desprenden dos formas de reparación económica que han producido un conflicto de equidad entre ambos grupos de víctimas y sus respectivos familiares sobrevivientes con el Estado de Chile; en el caso en que la víctima estaba fallecida al momento de ser reconocida por el Estado, pese a que se cumplió con todos los requisitos exigidos para ser considerada como tal, la restitución del derecho a la indemnización justa fue significativamente menor. Esta situación produjo efectos retraumatizantes y fuertes rechazos en familiares y agrupaciones ${ }^{23}$. Las inequidades que generan las medidas de reparación del Estado son una muestra del efecto deletéreo y revictimizante que producen sobre las personas que se pretende beneficiar, no siempre contribuyen a proteger la salud y la calidad de vida de los afectados sino, por el contrario, se transforman en factores de riesgo para su salud, como es el caso de los familiares de fallecidos antes del año 2004 (34,2\% del total).

Considerando los efectos nocivos de la traumatización extrema sobre los procesos psicológicos, la ausencia de estrategias de prevención en salud a nivel de las políticas públicas de salud genera inequidad. Respecto de las inequidades en salud, OMS dice: "La prueba crucial para saber si las dife- rencias en salud resultantes son consideradas injustas parece depender en mayor medida de si la gente eligió la situación que causó la mala salud o si ello estuvo fundamentalmente fuera de su control directo"24. Esta aseveración tiene un máximo sentido para la población en estudio, que estuvo afectada por un continuum de experiencias de traumatización extrema (tortura, exilio, ejecución, desaparición, etc.) durante 17 años de dictadura, provocando una serie de secuelas biopsicosociales derivadas de estas experiencias, las que fueron agravadas por el contexto de impunidad, gatillando procesos de retraumatización ${ }^{25,26}$. El propio informe de la CNPPT refiere que el acceso a la reparación en salud de las víctimas ha sido tardío y las víctimas reclaman la poca cobertura que tiene el PRAIS para atender sus necesidades.

Considerando lo señalado anteriormente, sugerimos algunas estrategias necesarias para mejorar la política de reparación en salud:

1. Los resultados de este estudio pueden servir para que PRAIS desarrolle estrategias de prevención, diagnóstico y tratamiento en víctimas de la dicadura cívico-militar.

2. Que el Estado de Chile fortalezca la perspectiva intersectorial en los procesos de reparación integral en salud, esto es, que se mejoren los recursos económicos, humanos y técnicos para la solución en concreto de las necesidades señaladas por las víctimas.

3. Para tales fines es urgente una reformulación global del mecanismo financiero de PRAIS, su desfinanciamiento actual impide una diversificación y extensión de las tareas sanitarias. Un programa de estas características debe ser analizado desde una óptica diferencial respecto de los programas tradicionales del sistema público de salud, así lo han entendido instituciones internacionales como la OMS y en el plano nacional la propia Norma Técnica Ministerial de PRAIS, que señala: "Que la atención reparatoria en salud tiene su base en el reconocimiento del daño $y / o$ el riesgo aumentado (...) debido a que la tensión permanente a la que estas personas han estado sometidas, las ha hecho más vulnerables a enfermar"27.

4. Finalmente, las comisiones de reconocimiento de víctimas deben ser una instancia permanente del Estado de Chile y diseñadas con una perspectiva de género con el objeto facilitar el reconocimiento de las mujeres y de ciudadanos 
que por muy diversas razones viven el efecto retraumatizante de no haber logrado validar hasta hoy sus testimonios de tortura.

\section{Referencias}

1. Koupil I, Shestov DB, Sparén P, Plavinskaja S, Parfenova $\mathrm{N}$, Vågerö D. Blood pressure, hypertension and mortality from circulatory disease in men and women who survived the siege of Leningrad. Eur J Epidemiol 2007; 22 (4): 223-34. doi:10.1007/s10654-007-9113-6.

2. Bramsen I, Deeg DJH, van der Ploeg E, Fransman S. Wartime stressors and mental health symptoms as predictors of late-life mortality in World War II survivors. J Affect Disord 2007; 103 (1-3): 121-9. doi:10.1016/j. jad.2007.01.014.

3. Page WF, Brass LM. Long-Term Heart Disease and Stroke Mortality among Former American Prisoners of War of World War II and the Korean Conflict: Results of a 50-Year Follow-Up. Mil Med 2001; 166 (9): 803-8. doi:10.1093/milmed/166.9.803.

4. Solomon Z, Greene T, Ein-Dor T, Zerach G, Benyamini Y, Ohry A. The long-term implications of war captivity for mortality and health. J Behav Med 2014; 37 (5): 84959. doi:10.1007/s10865-013-9544-3.

5. Den Velde WO, Deeg DJH, Hovens JE, van Duijn MAJ, Aarts PGH. War Stress and Late-Life Mortality in World War II Male Civilian Resistance Veterans. Psychol Rep 2011; 108 (2): 437-48. doi:10.2466/02.10.16. PR0.108.2.437-448.

6. Williams RL, Medalie JH, Zyzanski SJ, Flocke SA, Yaari S, Goldbourt U. Long-term mortality of Nazi concentration camp survivors. J Clin Epidemiol 1993; 46 (6): 573-5.

7. Fund N, Ash N, Porath A, Shalev V, Koren G. Comparison of Mortality and Comorbidity Rates Between Holocaust Survivors and Individuals in the General Population in Israel. JAMA Netw Open 2019; 2 (1): e186643-e186643. doi:10.1001/jamanetworkopen.2018.6643.

8. Bercovich E, Keinan-Boker L, Shasha SM. Long-Term Health Effects in Adults Born during the Holocaust. 2014; 16: 5 .

9. Madariaga, C. Monografía: Trauma Psicosocial, Trastorno de Estrés Postraumático y Tortura. Santiago, Chile: Editorial CINTRAS; 2003. p. 9-11.

10. Becker D, Castillo MI, Gómez E, Kovalskys J, Lira E. Psicopatología y proceso psicoterapéutico de situaciones políticas traumáticas. En: Martín-Baró (ed.), Psicología social de la guerra: trauma y terapia. San Salvador Editorial UCA; 1990.
11. Ministerio del Interior. Informe de La Comisión Nacional Sobre Prisión Política y Tortura. Ministerio del Interior. Santiago, Chile; 2005.

12. Ministerio Secretaría General de la Presidencia. Comisión Asesora para la calificación de Detenidos Desaparecidos, Ejecutados Políticos y Víctimas de Prisión Política y Tortura. 2011. Disponible en:https://www. indh.cl/bb/wp-content/uploads/2017/01/Informe2011. pdf [Consultado el 2 de abril de 2020].

13. Krug EG, Dahlberg LL, Mercy JA, Zwi AB. Informe mundial sobre la violencia y la salud. Revista do Instituto de Medicina Tropical de Sao Paulo. 2003; 45 (3).

14. Larizgoitia I, Izarzugaza I, Iraurgi I, Ballesteros J, Forero CG, Markez I, et al. Impacto de la violencia colectiva en la salud. Resultados del estudio ISAVIC en el País Vasco. Gaceta sanitaria 2011; 25 (2): 108-14

15. Dubordieu M, Nasi ML. Cáncer y Psico-neuro-inmunología. Clínica integrativa en Oncología. Argentina: Nativa Editorial; 2017: 46- 65

16. Guajardo G. Suicidios contemporáneos. Vínculos, desigualdades y trasformaciones socioculturales. Ensayos sobre violencia, cultura y sentido. Santiago, Chile: Ediciones FLACSO; 2017. p. 33.

17. Gobierno de Chile. Ministerio de Salud. Departamento de Estadísticas e Información en salud (DEIS). Disponible en: https://www.deis.cl. [Consultado el 15 de febrero de 2020].

18. Aguilar, Diana Patricia Morales. Desafíos en psicoterapia: trauma complejo, apego y disociación. Avances en Psicología 2018; 26 (2): 135-44. Disponible en http:// revistas.unife.edu.pe/index.php/avancesenpsicologia/ article/view/1186 [Consultado el 10 de octubre de 2020].

19. Escribens, P. Proyecto de vida de mujeres víctimas de violencia sexual en conflicto armado interno. Estudio para la Defensa de los Derechos de la Mujer DEMUS 2012: 30-40. Disponible en http://bvk.bnp.gob.pe/ad$\mathrm{min} /$ files/libros/730_digitalizacion.pdf [Consultado el 8 de julio de 2020].

20. Álvarez, V. Género y violencia: Memorias de la represión sobre los cuerpos de las mujeres durante la última dictadura militar argentina. Nomadías 2019; 19 (7): 63-83. doi: 10.5354/0719-0905.2015.36763.

21. Maldonado Garay, J. (2019). Violencia política sexual: una conceptualización necesaria. Nomadías 2019; 27 (7): 143-66. doi: 10.5354/0719-0905.2019.54379.

22. Women's Link Worldwide. La Verdad en voz alta. Extractos de la Sentencia "El Pueblo de la República de Guatemala contra José Efraínn Rios Montt. 2017. p. 3644.Disponible en https://www.womenslinkworldwide. org/files/2959/la-verdad-en-voz-alta.pdf [Consultado el 15 de marzo de 2020]. 
23. Ex-Prisioneros Políticos de Chile, Comando Unitario de Ex-Prisioneros Políticos y Familiares, Coordinadora Nacional de Ex- Presos Políticos Salvador Allende. Carta abierta para Ministro del Interior Don Mario Fernández 2015 Disponible en https://www.camara.cl/verDoc. aspx?prmTIPO=DOCUMENTOCOMUNICACIONCUENTA\&prmID=18205 [Consultado el 18 de mayo de 2020].

24. Whitehead M. The concepts and principles of equity and health. Copenhagen: WHO Regional Office for Europe (EURO); 1990.

25. Madariaga C, Brinkmann B. Del cuerpo y sus sucesivas muertes: identidad y retraumatización. Particularidades del proceso de exhumaciones vivido en Chile. Santiago:
Centro de Salud Mental y Derechos Humanos (CINTRAS), 2006. Disponible en http://cintras.org/textos/ monografias/Monografia12.pdf [Consultado el 5 de octubre de 2020].

26. Madariaga C. Retraumatización: hacia una conceptualización necesaria. Reflexión 2006; 32: 4-8. Disponible en http://www.cintras.org/textos/reflexion/r32/retraumatizacion.pdf [Consultado el 05 de octubre de 2020].

27. Ministerio de Salud. Norma Técnica para la Atención de Personas Afectadas por la Represión Política ejercida por el Estado en el período 1973- 1990. 2014 p.7. Disponible en http://prais.redsalud.gob.cl/wrdprss_minsal/ wp-content/uploads/2016/08/Norma-Técnica.pdf [Consultado el 22 de marzo de 2020]. 